\title{
Role of short-range atom-atom forces in formation of the orientational structure of simple molecular crystals
}

\author{
E.S. Yakub \\ Odessa National Economic University, 8 Preobrazhenskaya, Odessa 65082, Ukraine \\ E-mail: eugene.yakub@gmail.com
}

Received October 24, 2018

\begin{abstract}
An attempt is made to explain the appearance of certain phases having different orientational and spatial structures in the phase diagram of crystals formed by tetrahedral molecules. The classical Monte Carlo method is applied to solid heavy methane $\mathrm{CD}_{4}$ and the role of various contributions to the non-central intermolecular interactions in formation of the orientational structure in simple molecular crystals is assessed.
\end{abstract}

Keywords: phase diagram, Monte Carlo simulation, heavy methane, molecular rotation, atom-atom interactions.

\section{Introduction}

An extensive literature is devoted to crystals formed by simple molecules and to explaining the structural complexity of their solid-state phase diagram [1].

Despite the essential progress of modern quantummechanical $a b$ initio methods which can now provide rather accurate potential energy surfaces [2] and allow reliable predictions of phase diagrams in the region of extreme temperatures and pressures, they still cannot explain appearance of low-temperature phases having different spatial and orientational structures and are unable answer the question of what types of intermolecular interactions are responsible for the appearance and location of a particular phase on it, what is the role of quantum effects etc.

Even methane $\mathrm{CH}_{4}$ having simplest molecular structure has very complex phase diagram, where phases differ in both spatial and orientational structure. Special attention of researchers was paid to the phase II, which has unusual orientational structure. It has been established for a long time that in phase II existing both in $\mathrm{CH}_{4}$ and $\mathrm{CD}_{4}$ [3] solids in a narrow low-temperature range and only at relatively low-pressures part of molecules are orientationally disordered when the rest is spatially oriented.

According to Press [4] six of eight molecules in phase II order with a certain local (cubic) symmetry, while the remaining two are orientationally disordered. Later, at pressures above $5 \mathrm{kbar}$, a metastability region and phase IV were discovered [5]. James and Keenan [6] developed the first theoretical model of phase II in $\mathrm{CD}_{4}$ which accounts only for one type of intermolecular interactions, namely the octupole-octupole forces acting between nearest neighbors fixed in sites of a static face-centered cubic (fcc) lattice.
The purpose of this paper is to elucidate the role of another type of forces, namely atom-atom interactions, in the formation of low-temperature phases in molecular crystals. We restrict ourselves to considering the simplest type of non-spherical molecules, namely, tetrahedral, and use the classical Monte Carlo method. Additional methods needed to describe the rotational degrees of freedom and orientational ordering of tetrahedral molecules have been developed and applied in the computer simulation. Heavy methane $\mathrm{CD}_{4}$ was chosen as an object of study to minimize the influence of quantum effects. Particular attention is paid to the formation of phase II, which has the above-mentioned unusual orientational structure.

Molecular interaction models used in simulations are described in the next section. The basic simulation results are presented in Sec. 3. In Sec. 4 these results are discussed and compared with existing theoretical approaches and experimental data. A sensitivity study is also presented here. In the last section, we provide general conclusions and outline ways to solve the problems that remain unresolved within the current approach.

\section{Molecular interaction model}

The simplest model represents interaction energy of two molecules as a sum of central and non-central octupoleoctupole $(\Omega-\Omega)$ interactions

$$
\Phi_{i j}=\Phi_{\mathrm{CC}}\left(r_{i j}\right)+u_{\Omega-\Omega}\left(r_{i j}, \boldsymbol{\omega}_{i}, \boldsymbol{\omega}_{j}\right)
$$

and is based on the hypothesis of pair additivity of intermolecular interactions $U_{N}=\sum_{0<i<j \leq N} \Phi_{i j}$. 
Here $\boldsymbol{\omega}_{i}=\{\theta, \varphi, \psi\}$ determines the orientation of the tetrahedral molecule defined by three Eulerian angles $\theta, \varphi$, and $\psi$. The $\Omega-\Omega$ interactions decay with 7 th degree of the reciprocal intermolecular distance $r_{i j}$. Their explicit orientational dependence is well known [7]. The central $\Phi_{\mathrm{CC}}\left(r_{i j}\right)$ interactions are as usual described by the Lennard-Jones (12-6) potential. This model was successfully applied recently to the fcc phase of methane at elevated temperatures [8].

In this study we use a more advanced model including two additional types of short-range atom-atom interactions of heavy methane molecules:

$$
\begin{aligned}
\Phi_{i j}= & \Phi_{\mathrm{CC}}\left(r_{i j}\right)+\sum_{1 \leq k \leq 4}\left(\Phi_{\mathrm{CD}}\left(r_{i, j k}\right)+\Phi_{\mathrm{CD}}\left(r_{j, i k}\right)\right)+ \\
& +\sum_{1 \leq k, l \leq 4} \Phi_{\mathrm{DD}}\left(r_{i k, j l}\right)+u_{\Omega-\Omega}\left(r_{i j}, \boldsymbol{\omega}_{i}, \boldsymbol{\omega}_{j}\right) .
\end{aligned}
$$

Here $r_{i, j k}$ are distances between carbon atom of $i$ th molecule and $k$ th $(k=1, \ldots, 4)$ deuterium atom of $j$ th molecule. $\mathrm{C}-\mathrm{D}$ bonds are considered to be rigid, and their length fixed at $L=1.095 \AA$.

Such model much better reflects the nature of the shortrange interactions in $\mathrm{CD}_{4}$ solid, but its calibration is more complicated. For the practical application of this model in addition to the octupole moment at least two potential parameters are required for each type $(\mathrm{C}-\mathrm{C}, \mathrm{C}-\mathrm{D}$ and $\mathrm{D}-\mathrm{D})$ of atom-atom interaction.

To reduce the number of model parameters the usual Lorentz-Berthelot combination rules were adopted:

$$
\sigma_{\mathrm{CD}}=1 / 2\left(\sigma_{\mathrm{CC}}+\sigma_{\mathrm{DD}}\right), \quad \varepsilon_{\mathrm{CD}}=\sqrt{\varepsilon_{\mathrm{CC}} \varepsilon_{\mathrm{DD}}} .
$$

Here $\sigma_{\mathrm{DD}}$ and $\varepsilon_{\mathrm{DD}}$ are parameters of the Lennard-Jones (12-6) potential approximating atom-atom interaction energy of two hydrogen atoms. We accepted values $\sigma_{\mathrm{DD}}=$ $=2.81 \AA$ and $\varepsilon_{\mathrm{DD}} / k_{B}=8.6 \mathrm{~K}$ used in the recent simulations of El-Sheikh et al. [9], because they provide an excellent approximation for non-empirical potential of non-valent interaction between two hydrogen atoms used earlier in our computer simulations of hydrogen and deuterium fluids [10].

For the octupole moment it was also accepted the same value $\left(\Omega=2.3 \cdot 10^{-34}\right.$ esu) as in Ref. 9. This value is close to that accepted by James and Keenan [6] but essentially differs from the value $\left(\Omega=4.5 \cdot 10^{-34} \mathrm{esu}\right)$ recommended in [1] and accepted in [8].

The last value provides reasonable results only within the framework of the simplest interaction model (1) because it effectively represents contributions of all other types of short-range non-central forces acting between methane molecules which are ignored in this model.

Two remaining parameters $\left(\sigma_{\mathrm{CC}}\right.$ and $\left.\varepsilon_{\mathrm{CC}}\right)$ were fitted to reproduce the low-temperature density of the $\mathrm{CD}_{4}$ solid. The whole set of parameters adopted in this work is shown in Table 1 .
Table 1. Adopted parameters of the intermolecular interaction potential (2)

\begin{tabular}{c|c}
\hline \hline Parameter & Value \\
\hline \hline$\varepsilon_{\mathrm{DD}} / k_{B}$ & $8.6 \mathrm{~K}$ \\
\hline$\sigma_{\mathrm{DD}}$ & $2.8 \AA$ \\
\hline$\varepsilon_{\mathrm{CC}} / k_{B}$ & $50 \mathrm{~K}$ \\
\hline$\sigma_{\mathrm{CC}}$ & $3.63 \AA$ \\
\hline$\Omega$ & $2.3 \cdot 10^{-34} \mathrm{esu}$ \\
\hline \hline
\end{tabular}

The pairwise interaction energy (2) parameterized accordingly to Table 1 and pre-averaged over all orientations is in reasonable agreement with the central Lennard-Jones (12-6) potential recommended in the monograph of Manzhelii et al. [1] as shown in Fig. 1.

\section{Computer simulation}

The adopted potential model (2) was applied in study of structure and thermodynamic properties of crystalline $\mathrm{CD}_{4}$ using Monte Carlo simulation method in NVT ensemble.

Simulation processes was performed in cubic main cell containing $N$ molecules with usual periodic boundary conditions and include equilibration and statistics collection periods.

Each Monte Carlo step consisted of:

- random choice of a molecule to move;

- random displacement of its center of mass;

- random rotation of the molecule;

- application of the Metropolis rejection rule etc. [11].

A number of Metropolis Monte Carlo simulations of crystalline $\mathrm{CD}_{4}$ were performed at temperatures from 10 up to $80 \mathrm{~K}$ and at densities, corresponding to pressures up

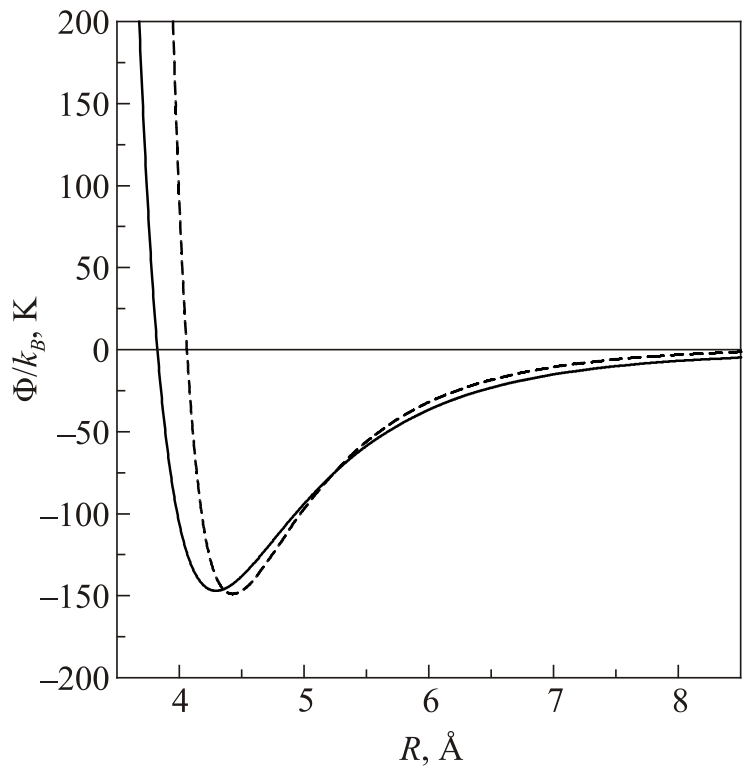

Fig. 1. Comparison of the pre-averaged pair interaction energy Eq. (2) (dashed line) with the central Lennard-Jones (12-6) potential $\left(\varepsilon / k_{B}=148 \mathrm{~K}, \sigma=3.817 \AA\right)[1]$ (solid line). 
to $1 \mathrm{GPa}$. Cubic cells containing $N$ molecules placed in the sites of a fcc lattice were created at a start. The initial orientations of all molecules were obtained by minimizing the potential energy of the lattice.

Two different sizes of the main Monte Carlo cell were used: a "small" cell $N=32$ (160 atoms), and a "large" cell, having twice the size of a "small" cell and containing $N=$ $=256$ molecules (1280 atoms). Intermolecular potentials were "cut" at $1 / 2$ of the cell size $\left(R_{\text {cut }} \sim 5 \AA\right.$ in the "small" cell and $R_{\text {cut }} \sim 10 \AA$ in the "large" cell). Therefore the structure of the "small" cell was formed mainly by the shortrange forces acting between the nearest neighboring atoms.

Two types of the initial conditions in main cell were used:

(a) "large" cell composed from eight relaxed "small" cells;

(b) "large" cell with fcc spatial structure and molecular orientations minimizing the potential energy of the lattice.

In the case (a) the "large" cell at the start of the new simulation is filled by eight copies of a "small" 32-molecule cell previously relaxed in a separate Monte Carlo run. In this case the relaxation period of the "large" cell was essentially shorter and this type of initial conditions was used throughout.

Sometimes the results of two runs with different initial conditions were the same, but sometimes the structure inherited from the "small" cell led to another stable spatial and orientational structure of the "large" cell and the final states have slightly lower energy and pressure. Below we discuss such cases in connection with the problem of metastability.

The orientational structure was studied in two ways:

- via monitoring the numbers of the events of "rotation"* for each molecule;

- by calculating orientational distributions of chemical bonds.

The event of the molecular "rotation" within Monte Carlo procedure was treated as a change of the sign of any projection $(x, y$, or $z)$ of the molecular orientation vector in a single accepted Monte Carlo step ${ }^{* *}$. When the frequency of such "rotations" of the molecule reaches certain minimum limit (i.e., such an event is not accidental), this molecule was regarded as "rotating".

Monte Carlo simulations started at relatively high temperatures and low pressures, were repeated with the step by step increasing density and the changes appearing in the spatial and orientational structures of the solid were monitored.

\section{Results and discussion}

\subsection{Spatial structure}

At $T>50 \mathrm{~K}$ and at relatively low densities the initial fcc distribution of molecular centers survives in both "small"

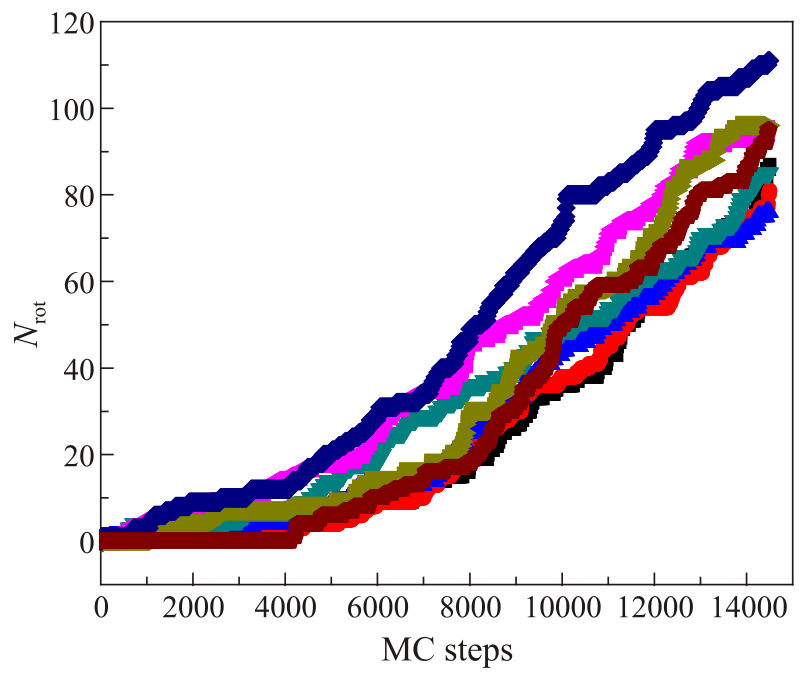

Fig. 2. (Color online) Numbers of molecular "rotations" at different lattice positions vs. number of successful steps per molecule in phase I $\left(T=40 \mathrm{~K}, V=31.75 \mathrm{~cm}^{3} / \mathrm{mole}\right)$.

and "large" cells, and a pronounced effect of molecular "rotations" characteristic to phase I was observed. A typical case is shown in Fig. 2.

When the density increases at a fixed temperature the slope of the curves presented in Fig. 2 decreases and at a certain density the molecular "rotation" stopped. We interpret this effect as a transition to the phase III.

Special attention was paid to the formation of structures which could be identified as corresponding to the phase II. With a decrease in temperature, noticeable changes appear not only in the orientational but also in the spatial structure of solid. These changes appear first and were more pronounced in "small" cells. Centers of molecules begin displace from their initial fcc positions in such a way that instead of four, eight different lattice positions are formed.

Only two of these eight lattice positions correspond to molecules which continue "rotate" (Fig. 3). Such states were interpreted as belonging to the phase II.

Displacements of molecular centers appearing in such states are presented in Fig. 4 and in Table 2. In the first lattice position occupied by molecules marked in Fig. 4 as $1,13,21$, and 25 molecular centers are displaced along $X$ axis from their initial positions in the fcc lattice. Molecules $2,22,14,26$ belonging to the second lattice position are relocated along $Y$ axis, and molecules 3, 15, 23, and 27 (third lattice position) moved along $Z$ axis. Molecules which belong to the lattice positions 5, 6 and 7 (see Table 2) displace correspondingly along the same axes in the opposite direction.

Centers of the remaining eight molecules occupying lattice positions 4 and 8 are displaced along the main diagonal

* Here and below, we use quotation marks to emphasize the conventionality of this term within the Monte Carlo method used.

** The maximum possible angular displacement step was retained low enough to avoid the jump between the two regions, in fact separated by high potential barriers insurmountable for molecules in their real dynamics. 


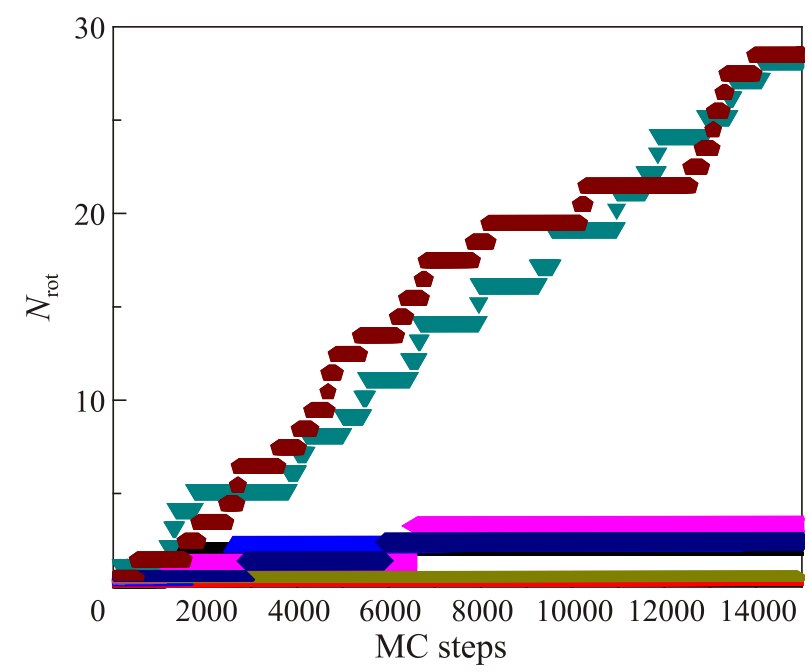

Fig. 3. (Color online) Numbers of molecular "rotations" at different lattice positions vs. number of successful steps per molecule at $T=30 \mathrm{~K}$ and $V=30 \mathrm{~cm}^{3} /$ mole (phase II).

of the cubic cell. As a result, molecular centers form a new cubic lattice which is alike to that discovered by Press [4] in phase II.

Table 2. Directions of molecular centers displacements from the initial fcc sites for different lattice positions in phase II

\begin{tabular}{c|c|c}
\hline \hline Lattice position & Molecules & Displaced along \\
\hline \hline 1 & $1,13,21,25$ & $+X$ \\
\hline 2 & $2,22,14,26$ & $+Y$ \\
\hline 3 & $3,15,23,27$ & $+Z$ \\
\hline 4 & $4,16,24,28$ & $+X Y Z$ \\
\hline 5 & $5,9,17,29$ & $-X$ \\
\hline 6 & $6,10,18,30$ & $-Y$ \\
\hline 7 & $7,11,19,31$ & $-Z$ \\
\hline 8 & $8,12,20,32$ & $-X Y Z$ \\
\hline \hline
\end{tabular}

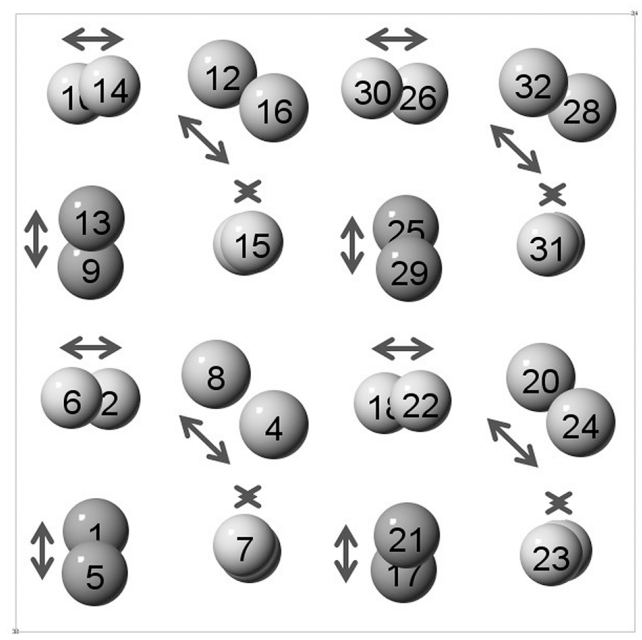

Fig. 4. Distortion of a fcc lattice induced by short-range atomatom forces in a "small" Monte Carlo cell.

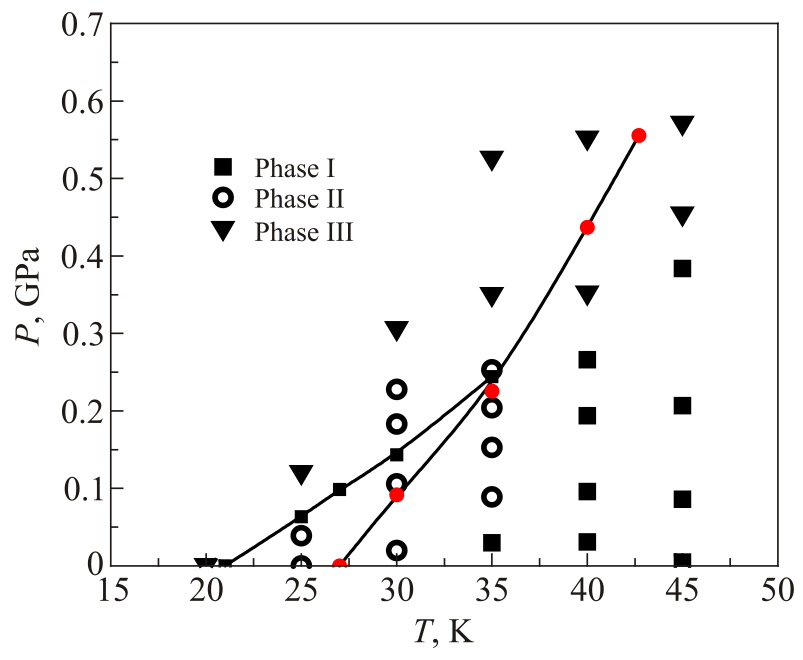

Fig. 5. Comparison of the predicted low-temperature phase diagram of $\mathrm{CD}_{4}$ with the experimental data of van der Putten [5] (solid lines).

Such structure of the "small" cell when transferred to the "large" one according to the initial conditions of type (a) mentioned above may survive there or may be destroyed by the interaction of more distant molecules. In the first case we obtain an orientational structure which resembles that proposed for phase II: the "rotating" molecules in the "large" cell are only those belonging to the lattice positions 4 and 8 (with molecular centers displaced along the diagonal of the cell). This case was interpreted as corresponding to the formation of phase II and is illustrated in Fig. 4.

Results of the whole set of the Monte Carlo simulations performed are summarized in Fig. 5. States where all molecules are "rotating" (phase I) are marked by circles. Triangles denote such states, where only a quarter of all molecules "rotate" as in Fig. 4 (phase II). And squares represent such states where no systematic "rotations" were found (phase III). As it can be seen, there is a qualitative agreement with the experimental data of van der Putten [5]. The deviations are of the same order as between data of van der Putten [5] and of Stewart [3].

\subsection{Orientational structure}

Due to appearance of states with specific spatial structures attributed to phase II, special attention was paid to a more detailed study of their orientational structure. Angular distributions of C-D chemical bonds directions of molecules occupying different lattice positions were evaluated. Their analysis may help to understand the nature of molecular "rotations" or "librations" and provide answers to such questions as: does it really exist "free rotation" of $\mathrm{CD}_{4}$ molecules in phase I, does it really correspond to the uniform angular distribution of chemical bonds etc.?

The angular distribution the of the bonds directions in phase III is the simplest one and is illustrated in Fig. 6. It can be seen that there exist only four highly preferred di- 


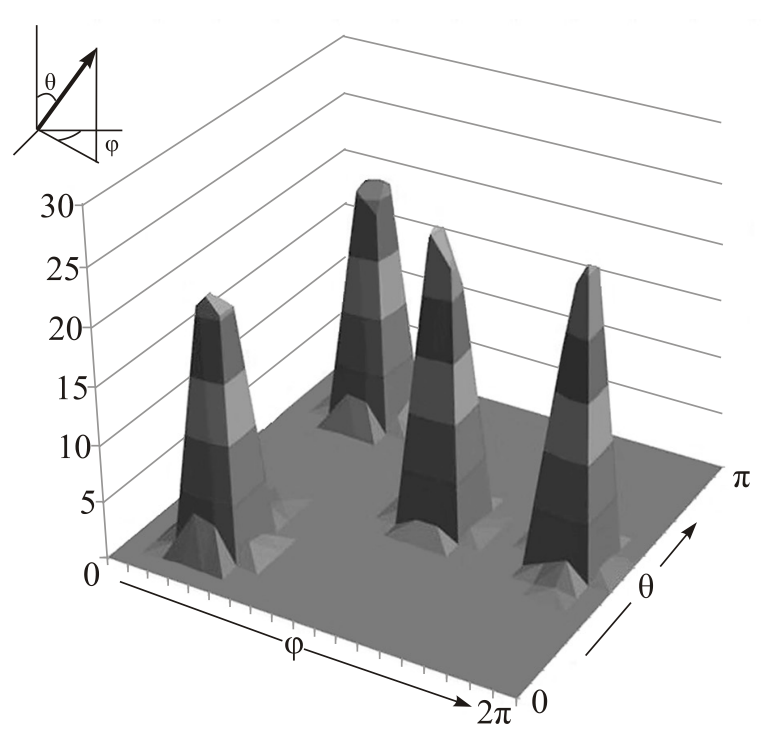

Fig. 6. Low-temperature angular distributions of chemical bonds directions in phase III $(T=10 \mathrm{~K})$.

rections corresponding to the four chemical bonds which can only slightly deviate from these preferred directions.

The same distributions corresponding to the states attributed to phase II are shown in Fig. 7. As can be seen from Fig. 7(a), in contrast to phase III the angular distribution of chemical bonds of "non-rotating" molecules has eight pronounced peaks. That means each bond has two preferred directions and each "non-rotating" molecule librates between two available orientations.

The "rotating" molecules, belonging to lattice sites 4 and 8 (Table 2), have a very different angular distribution presented in Fig. 7(b). It has one broad and several narrow peaks which are interconnected by regions having smaller but non-zero probability density. These regions allow transitions between different peaks and provide mechanism of

(a)

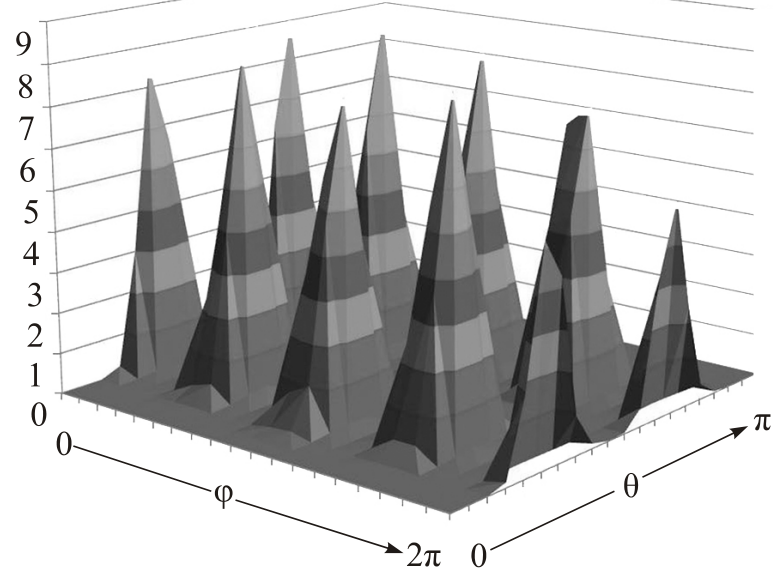

molecular "rotations". At the same time it is clear that these "rotations" are extremely hindered.

At low temperatures a similar picture is also observed in phase I. All molecules are "rotating" but their rotations remain highly hindered. As can be seen in Fig. 8, with increasing temperature the orientational distribution is approaching to that which is characteristic for free rotation. The only strong correlation remaining here between the bonds directions is the intramolecular one.

\subsection{Sensitivity study}

It is clear that the results described above may be sensitive both to the model parameters and to some details of the simulation procedure adopted. Therefore a number of additional simulations aiming to reveal the sensitivity of the results to the model parameters and to the initial conditions used in simulation was carried out.

First we studied the sensitivity of the obtained results to the change in the parameters of the model. The purpose of these tests was to assess the role of short-range atom-atom forces in the formation of the orientational structure. All the simulations were repeated using the simplest model of interaction (1), including only the central forces represented by the Lennard-Jones (12-6) potential shown by solid line in Fig. 1, and the octupole-octupole contribution.

The result was as follows: molecular "rotations" existed at any value of the octupole moment (up to $\Omega=4.5 \cdot 10^{-34}$ esu [1]) but their intensity (their number per successful Monte Carlo step) decreases rapidly with decreasing temperature and increasing density. No signs of the molecular centers displacements and states where only part of molecules "rotate", characteristic for phase II (Table 2) were found.

Analysis of results obtained with the simple molecular model (1) shows that when temperature decreases, no displacements of molecular centers, similar to that shown in Fig. 4 appear, and in this case no molecules stop "rotating"

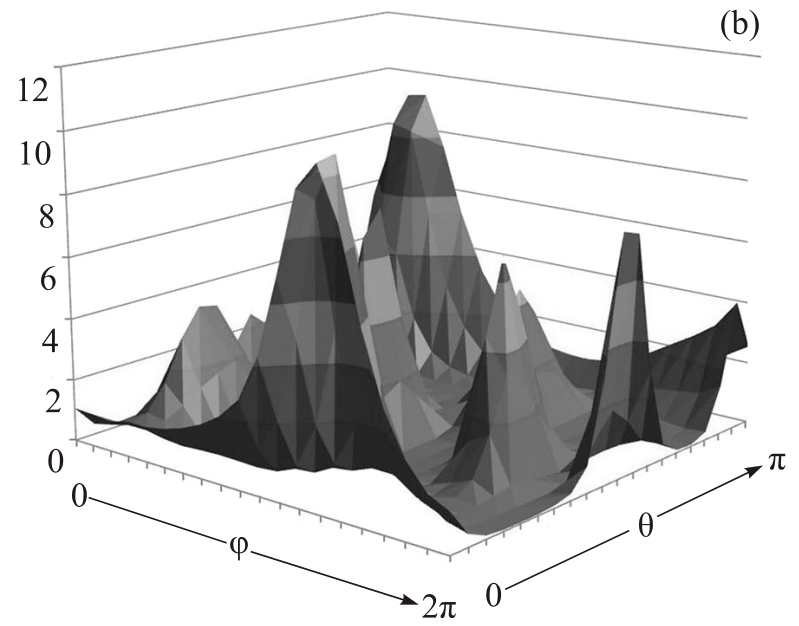

Fig. 7. Angular distributions of chemical bonds directions in phase II $(T=30 \mathrm{~K})$ : (a) "librating" molecules at lattice sites 2 and 6 ; (b) "rotating" molecules at lattice sites 4 and 8 (see Table 2). 


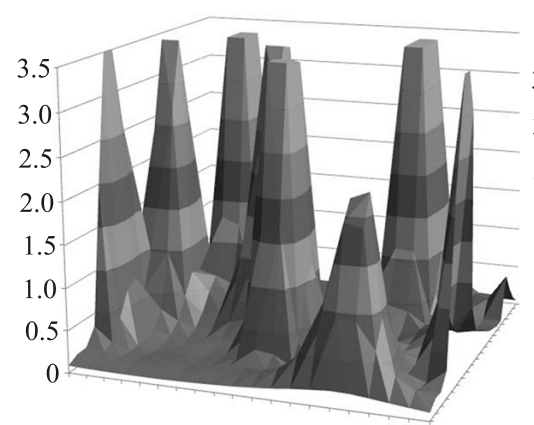

$T=40 \mathrm{~K}$

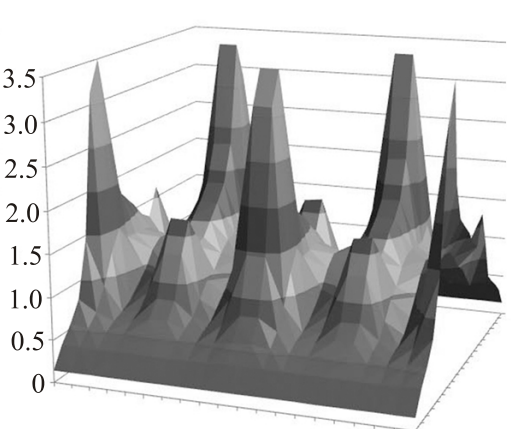

$T=60 \mathrm{~K}$

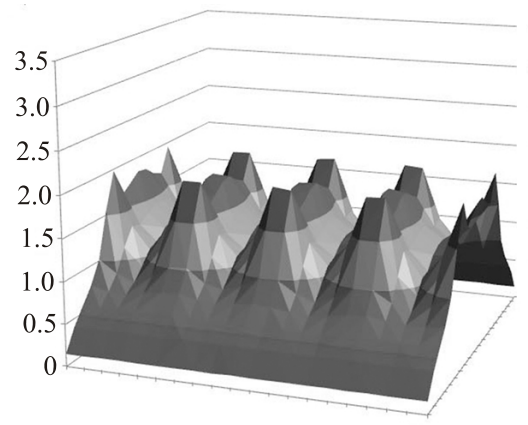

$T=80 \mathrm{~K}$

Fig. 8. Angular distributions of C-D bonds directions in phase I at different temperatures on isochore $V=30 \mathrm{~cm}^{3} / \mathrm{mole}$. Location of the coordinate axes the same as in Fig. 7.

at once and even the transition to states without "rotation", which could be attributed to phase III, appear gradually. Within the simulation method applied the exact location of the transition from phase I to phase III cannot be determined but the absence of structures resembling phase II where only part of molecules are "rotating", was evident.

This contradicts the results of James and Keenan [6], who found signs of phase II using actually the same model (1) and assuming that the centers of all molecules are fixed at the fcc lattice sites.

\subsection{Metastability}

The second set of tests was performed by changing the initial conditions of the Monte Carlo simulations. Instead of the mentioned above standard condition: (a) with the "large" 256-molecule cell initially build from eight equilibrated "small" cells, we tested another type of initial crystalline structure: (b) with initial orientations of molecules minimizing the potential energy of the fcc lattice.

During these tests each Monte Carlo run with the "large" cell was performed at least twice, using these two different initial molecular configurations. Both at low and high densities the results were the same; spatial structure inherited in case (a) from "small" cells was slowly step-bystep re-created in the "large" cell in the case (b).

But at intermediate densities in the case (b) the initial fcc structure survives and led finally to another stable orientational structure in the "large" cell. This case usually corresponds to slightly higher values of both internal energy and pressure. A typical result of such double run is illustrated in Fig. 9 where internal energy on the isotherm is presented as a function of pressure for two different types of initial conditions for "large" cell: (a) and (b).

Definitely one of these states could be interpreted as a metastable one. Unfortunately the Metropolis Monte Carlo method applied here does not allow calculating Helmholtz free energy and we cannot state with confidence which of the obtained states is metastable. Nevertheless, the very fact of the appearance of a metastable state is beyond doubt. This is in line with the results reported by van der Putten [5] who discovered in the phase diagram of $\mathrm{CD}_{4}$ a broad area where such metastability take place. The darkened area in Fig. 9 corresponds to that the metastability region found in [5]. Its position on the phase diagram is not exactly the same as was found in our simulations but is in outline close to it.

\section{Conclusions}

Despite the fact that the Metropolis Monte Carlo method applied does not allow calculating free energy and, accordingly, determining the exact location of the phase transition lines in the phase diagram, it allows us to identify the role of the octupole-octupole and short-ranged atomatom forces in the formation of the spatial and orientational structure of simple molecular crystals.

Both octupole-octupole and short-ranged atom-atom forces are responsible for formation of low-temperature crystalline phases in molecular solids. Nevertheless the role

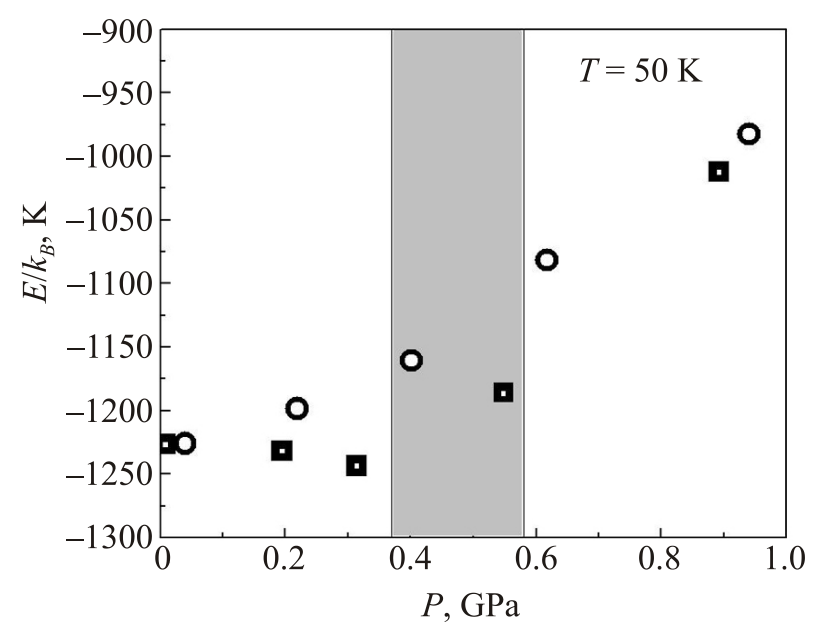

Fig. 9. Internal energy at $T=50 \mathrm{~K}$ as a function of pressure for two different types of initial conditions: "large" cell build from 8 relaxed 32-molecule "small" cells (squares); start from 256-molecule fcc lattice positions (circles). The darkened area corresponds to the metastability region found in [5]. 
of the short-range atom-atom repulsive forces is crucial in this respect.

As far as it possible to conclude on the basis of the present Monte Carlo study of solid heavy methane, shortrange atom-atom forces are responsible for the transition of the fcc phase I to the phase II. These forces determine formation of another spatial cubic structure $(F m 3 c)$, which in turn leads to formation of this specific orientational structure of phase II where only a one fourth of all molecules "rotate" and all other are librating around certain preferred orientations. It was found also that the same model allows reproducing the transition from orientationally disordered phase I to orientationally ordered phase III.

Angular distributions of the bonds directions predicted on the basis of the adopted molecular model and presented above in Figs. 6-9 allows us to conclude that molecular "rotations" in both orientationally disordered phase I and partially orientationally ordered phase II are highly hindered and became more or less "free" only near the melting point. This calls into question assessments carried out on the basis of the idea that "one-fourth of the molecules in phase II are free rotors" [12].

The results presented above show that the applicability of simple models accounting only for central intermolecular and octupole-octupole interaction of tetrahedral methane molecules is limited by the high-temperature region near the melting line [8] and does not reproduce transitions from orientationally disordered phase I to the phase II and to orientationally ordered phase III.

Sure, not all of the issues arising in the field of this study can be resolved on the basis of the classical Monte Carlo simulation approach. Many problems remaining are related to the limitations of the applied molecular model. Despite the fact that the atom-atom model has found its application for describing interactions in many molecular crystals [13], chemical bonds within this model are rigid and short-range atom-atom repulsion represented by Lennard-Jones (12-6) potentials is too stiff, which limits applicability of the model at high pressures [14]. Its application, for example, to methane, where quantum effects are more important [15], is limited, because it neglects these effects. Representation of the short-ranged electrostatic interaction only by the octupole-octupole forces is also limited.

Another problem worth to be noted in this context is the manifestation of the metastable states discussed above. Appearance of such states during transitions into other highpressure phases in many solids build from deuterated methane derivatives was discovered experimentally [12]. It is probably responsible for some other effects observed in such solids, e.g., for hysteresis of the thermal conductivity in a mixed $\mathrm{CD}_{4}-\mathrm{CH}_{4}$ observed at low pressure [16], what make this problem even more acute.

There remain also some much more general problems, such as the absence of a consistent theoretical method for estimating Helmholtz free energy of molecular solids and determining the location of phase boundaries between different crystalline phases. This problem was solved only for monatomic solids [17] by applying Mayer's group expansion technique for the Helmholtz free energy. The existence of preferred molecular orientations in all phases revealed in this work makes the extension of this approach to molecular crystals a promising direction in future theoretical studies.

1. V.G. Manzhelii, Structure and Thermodynamic Properties of Cryocrystals: Handbook, Begell House (1999).

2. A.V. Nikitin, M. Rey, and Vl.G. Tyuterev, J. Chem. Phys. 145, 114309 (2016).

3. J.W. Stewart, J. Phys. Chem. Solids 12, 122 (1959).

4. W. Press, J. Chem. Phys. 56, 2597 (1972).

5. D. van der Putten, N.J. Trappeniers, and K.O. Prins, Physica B 124, 193 (1984).

6. H.M. James and T.A. Keenan, J. Chem. Phys. 31, 12 (1969).

7. P. Isnard, D. Robert, and L. Galatry, Molec. Phys. 31, 789 (1976).

8. L. Yakub and E. Bodiul, J. Low Temp. Phys. 187, 33 (2017).

9. S.M. El-Sheikh, K. Barakat, and N.M. Salem, J. Chem. Phys. 124, 124517 (2006).

10. E.S. Yakub, Int. J. Thermophys. 22, 505 (2001).

11. R.Y. Rubinstein and D.P. Kroese, Simulation and the Monte Carlo Method, 3rd ed, Wiley Series in Probability and Statistics (2017).

12. D. van der Putten and K.O. Prins, Int. J. Thermophys. 10, 1205 (1989).

13. S.J. Stuart, A.B. Tutein, and J.A. Harrison, J. Chem. Phys. 112, 6472 (2000).

14. T.C. O'Connor, J. Andzelm, and M.O. Robbins, J. Chem. Phys. 142, 024903 (2015).

15. T. Yamamoto and Y. Kataoka, Phys. Rev. Lett. 20, 1 (1968).

16. A.I. Krivchikov, P. Stachowiak, E. Pisarska, and A. Jeżowski, Phys. Rev. B 75, 012303 (2007).

17. L. Yakub and E. Yakub, J. Chem. Phys. 136, 144508 (2012).

Роль короткодіючих атом-атомних сил у формуванні орієнтаційної структури простих молекулярних кристалів

$$
\text { Є.С. Якуб }
$$

Зроблено спробу пояснити причину появи деяких фаз, що мають різні орієнтаційні та просторові структури, на фазовій діаграмі кристалів, утворених тетраедричними молекулами. Класичний метод Монте-Карло застосовано до твердого важкого метану $\mathrm{CD}_{4}$ та оцінено роль різних внесків у нецентральні міжмолекулярні взаємодії в формування орієнтаційної структури простих молекулярних кристалів.

Ключові слова: фазова діаграма, метод Монте-Карло, важкий метан, молекулярне обертання, атом-атомні взаємодії. 


\section{Роль короткодействующих атом-атомных сил в формировании ориентационной структуры простых молекулярных кристаллов}

\section{Е.С. Якуб}

Сделана попытка объяснить причину появления некоторых фаз, имеющих разные ориентационные и пространственные структуры, на фазовой диаграмме кристаллов, образованных тетраэдрическими молекулами. Классический метод Монте-
Карло применен к твердому тяжелому метану $\mathrm{CD}_{4}$ и оценена роль различных вкладов в нецентральные межмолекулярные взаимодействия в формирование ориентационной структуры простых молекулярных кристаллов.

Ключевые слова: фазовая диаграмма, метод Монте-Карло, тяжелый метан, молекулярное вращение, атом-атомные взаимодействия. 